\title{
SALUD MENTAL DE LOS NIÑOS, NIÑAS Y ADOLESCENTES EN SITUACIÓN DE CONFINAMIENTO
}

Camilo Morales Retamal 


\section{CAMILO MORALES RETAMAL}

Psicólogo clínico infanto-juvenil. Magíster en Clínica Psicoanalítica con Niños y Jóvenes, Universidad Alberto Hurtado. Coordinador del Núcleo de Estudios Interdisciplinarios en Infancias de la Facultad de Ciencias Sociales, Universidad de Chile. 


\section{SALUD MENTAL DE LOS NIÑOS, NIÑAS Y ADOLESCENTES EN SITUACIÓN DE CONFINAMIENTO}

\section{INTRODUCCIÓN}

La pandemia global causada por el Covid-19 es una crisis sociosanitaria histórica que impactará en el bienestar y desarrollo de las sociedades a escala global, con profundos efectos económicos, sociales y políticos. Se trata de un hecho social (Durkheim, 1895) cuyas consecuencias abarcan múltiples dimensiones de la vida cotidiana, las relaciones sociales y la subjetividad. Es un acontecimiento que compromete lo individual y lo colectivo, que no escapa a las diferencias de género, etnia, localización geográfica o socioeconómicas. La magnitud de esta crisis es, en cierto punto, incalculable, y nos obliga a reflexionar sobre las implicancias que tendrá para las personas convivir con la vulnerabilidad y la incertidumbre, así como observar la producción de nuevas subjetividades en el campo de la vida cotidiana y la relación con el otro en la intimidad y en los espacios públicos y colectivos postpandemia.

Como señala el Comité de Derechos del Niño de Naciones Unidas (2020), niños, niñas y adolescentes (NNA) son una de las poblaciones más vulnerables en la pandemia, ya que están expuestos a graves efectos físicos, emocionales y psicológicos producto de las restricciones que significan el cierre de escuelas y jardines infantiles; pérdida del contacto con grupos de pares; limitaciones para el movimiento y actividades de recreación; y dificultades de acceso a contextos de protección frente a situaciones que amenacen sus derechos. Se calcula que cerca de 860 millones de NNA se verán afectados por las medidas de cuarentena (Orgilés et al., 2020).

Este nuevo escenario ha restringido el disfrute de ciertos derechos humanos como consecuencia de las medidas sanitarias que buscan prevenir y mitigar la propagación del virus en la población.

Por otro lado, los estados de emergencia y/o catástrofe, como el producido por el Covid-19, pueden mantenerse por largos periodos de tiempo y tener consecuencias a mediano y largo plazo en los procesos de aprendizaje, la salud mental, el aumento de la pobreza infantil y el riesgo de ser víctimas o testigos de violencia, entre otros graves problemas que pueden afectar el bienestar y desarrollo integral de la niñez (Unicef, 2020). 
Habida cuenta de lo anterior, el presente artículo tiene el propósito de describir y reflexionar sobre los principales efectos de la pandemia y el confinamiento en NNA, considerando las implicancias para su salud mental.

Se propone, en primer lugar, realizar una contextualización de la situación de la infancia en el contexto de pandemia y las principales dimensiones afectadas en este nuevo escenario global. En segundo lugar, se abordarán los efectos específicos de la experiencia de confinamiento de NNA teniendo a la vista algunas investigaciones que aportan información sobre los cambios de rutinas, la educación a distancia, nuevas formas de socialización y los vínculos afectivos. Finalmente, se propondrán algunas reflexiones que permitan articular los elementos presentados previamente con la importancia que tienen los vínculos afectivos en la protección de la salud mental infantil en un contexto de crisis sociosanitaria caracterizado por niveles significativos de aislamiento, vulnerabilidad e incertidumbre.

\section{LA VULNERABILIDAD DE LA NIÑEZ EN TIEMPOS DE PANDEMIA}

Pese a los significativos avances en materias de protección, salud y educación, la población infantil se enfrenta a un futuro incierto (Clark et al., 2019). Al cambio climático, la degradación ecológica, las poblaciones migratorias y las desigualdades generalizadas se suman los efectos de una pandemia global sin precedentes. Por lo tanto, la protección integral de los derechos de la niñez y el resguardo del futuro de nuevas generaciones dependerá de los esfuerzos que los gobiernos realicen para implementar políticas públicas que reconozcan a los NNA como sujetos de derechos y un grupo prioritario en el desarrollo sustentable de las sociedades.

Lo anterior cobra aún más relevancia frente a la situación de crisis sociosanitaria que afecta al mundo y particularmente a Chile. Organismos internacionales como el Comité de Derechos del Niño de Naciones Unidas (2020), Unicef (2020) y la Comisión Interamericana de Derechos de Humanos (2020) han expresado su preocupación por la situación de los NNA en todo el mundo, dada su condición de especial vulnerabilidad ante los efectos que producen los estados de emergencia y los confinamientos obligatorios y que se prolongan en el tiempo.

A su vez, la situación de emergencia implica visibilizar y reforzar los mecanismos de protección y participación tomando como directriz primordial el interés superior de NNA a través de medidas que consideren su nivel de desarrollo, trayectorias subjetivas, contextos comunitarios y vínculos afectivos. La protección de los derechos de la niñez en un contexto de crisis no puede ir en detrimento de los vínculos familiares y comunitarios (Cidh, 2020), como tampoco prescindir de instancias que permitan que NNA tengan la oportunidad de que sus opiniones sean 
escuchadas y tomadas en cuenta en aquellas decisiones que los involucran y afectan (Comité de Derechos del Niño, 2020).

Estas consideraciones resultan primordiales si se quiere, efectivamente, mitigar los potenciales efectos de un acontecimiento que impactará en prácticamente todas las dimensiones de la experiencia individual y colectiva de la infancia. Pero, además, son necesarias en el marco del respeto por los derechos de la niñez y los principios que sustentan el paradigma de la protección integral que reconoce a NNA como sujetos plenos y titulares de derechos y que establece una serie de obligaciones y estándares a los Estados para garantizar el bienestar y desarrollo de todo NNA (Beloff, 1999).

Lo anterior es fundamental dado que la situación de la infancia en el contexto de Covid-19 ha sido denominada como una "crisis global de los derechos de la niñez" (The Alliance for Child Protection in Humanitarian Action, 2020), ya que ha provocado trastornos significativos en las familias, las comunidades y las rutinas cotidianas. Estos efectos pueden observarse en tres niveles:

La pandemia de Covid-19 afecta a los niños de tres maneras principales: 1) por la infección con el propio virus, 2) por las repercusiones sociales y económicas de las medidas, como el control, la contención y la mitigación, destinadas a reducir o detener la transmisión del virus en diversos contextos, y 3) por los posibles efectos a largo plazo de la crisis, como la recesión económica y el retraso en los progresos hacia el logro de los Objetivos de Desarrollo Sostenible (ONU, 2020, p. 4).

La Organización de Naciones Unidas (2020) ha señalado que se trata de una crisis universal pues todos los niños, de todas las edades y de todos los países se verán afectados por las medidas de mitigación de la pandemia. Los efectos nocivos que se derivan de esta situación no se distribuirán de forma equitativa y muchos NNA se verán afectados de por vida si la gestión política de la crisis no considera las situaciones de vulnerabilidad en la que se encuentran muchos de ellos.

Según informes de organismos internacionales (HRW, 2020; ONU, 2020; Unesco, 2020; Unicef, 2020), es posible identificar cuatro ámbitos críticos en la infancia que serán afectados por la pandemia: educación, protección de derechos, pobreza y salud mental.

El cierre masivo de escuelas, colegios y jardines infantiles es un acontecimiento histórico que no tiene precedentes. Según la Unesco (2020), más de 1.500 millones de estudiantes de 188 países se han visto afectados por el cierre de escuelas debido al Covid-19, lo que representa más del 91\% de la población estudiantil mundial. 
Esta situación ha generado una interrupción en los procesos de aprendizaje y ha evidenciado además las brechas y desigualdades en el acceso a las plataformas digitales, disponibilidad de materiales para el aprendizaje en muchos centros educativos y un conjunto de condiciones adversas para desarrollar procesos educativos al interior de los hogares, como la falta de espacio físico o la ausencia del apoyo familiar para garantizar continuidad de los estudios. Para muchos niños, la crisis de Covid-19 significará una educación limitada o nula, un mayor retraso con respecto a sus compañeros e incluso el abandono escolar.

Las comunidades educativas son también esenciales en los procesos de socialización y en el establecimiento de una rutina que permita la organización de la vida cotidiana, por lo que los NNA afectados por el cierre de escuelas también perderán la sensación de estabilidad y las posibilidades de socialización que proporcionan estos espacios.

Otro de los ámbitos que presenta importantes desafíos en el contexto de pandemia son las situaciones que amenazan o vulneran los derechos de NNA. El estrés familiar que se produce por la pérdida del empleo, el aislamiento, el confinamiento excesivo y la ansiedad por la salud y las finanzas aumenta el riesgo de violencia al interior de la familia, entre la pareja o la ejercida por los cuidadores contra los niños (HRW, 2020). A su vez, las medidas de prevención de contagios pueden aumentar el número de casos de abuso infantil, ya que producen un corte abrupto de las relaciones seguras, positivas y de apoyo en las que NNA confían cuando se encuentran en dificultades, pero que dejan de estar disponibles por la cuarentena. Esto incluye la escuela, la familia extendida y la comunidad. Los derechos de los niños a la seguridad y la protección se ven amenazados en estas circunstancias:

El hogar debe ser la primera línea de defensa y protección del niño. Los factores estresantes relacionados con Covid-19 están amenazando esa defensa. Los hogares de todo el mundo están luchando para hacer frente a las nuevas restricciones de viaje y trabajo, las preocupaciones por la salud, la seguridad alimentaria, la inestabilidad financiera a nivel personal y mundial, y la información conflictiva sobre una serie de cuestiones (...) Todos y cada uno de estos factores pueden aumentar el riesgo de daño a los niños que ya están atrapados en situaciones de abuso y abandono. Estos factores también pueden aumentar la posibilidad de que los cuidadores excesivamente estresados se vuelvan violentos o abusivos. Estas nuevas tensiones se producen en un momento en que los niños son menos visibles para las personas y los profesionales que normalmente se dedican a su protección, y cuando los servicios de bienestar infantil y familiar 
están sobrecargados y desorganizados (Alianza para la Protección de la Infancia en la Acción Humanitaria, 2020, p. 2).

Un tercer factor de preocupación que da cuenta de la vulnerabilidad de los NNA es el probable aumento de la pobreza infantil. La recesión económica tendrá una serie de ramificaciones negativas a largo plazo e impactará en los niños. La pérdida de empleos en las familias con hijos hará crecer rápidamente la pobreza de este grupo de la sociedad.

Según un informe de Human Rights Watch (2020), los niños tienen más del doble de probabilidades de vivir en la pobreza que los adultos. A nivel mundial, aproximadamente uno de cada tres niños — cerca de 663 millones — viven en hogares que son multidimensionalmente pobres, lo que significa que carecen de servicios tan básicos como la nutrición o el agua potable.

En el caso de Chile, según datos de un informe de Unicef sobre los Objetivos de Desarrollo Sostenible en los países ricos (2017), un 25,5\% de los niños es pobre, lo que ubica a nuestro país último entre las naciones que pertenecen a la Ocde y la Unión Europea. Por lo tanto, es importante constatar la situación de vida previa a la crisis del Covid-19 y considerar que producto de la recesión económica esta cifra aumentará, con una serie de problemáticas asociadas.

Se estima que 385 millones de niños viven en la pobreza extrema (Unicef, 2016). Los bajos ingresos se asocian a mayores tasas de enfermedades crónicas, algunas de las cuales pueden aumentar el riesgo de enfermedades graves a causa de la pandemia. Las comunidades con bajos ingresos tienen más probabilidades de estar expuestas al contagio del virus, tienen tasas de mortalidad más altas, sufren económicamente y reciben una atención sanitaria de menor calidad.

En consecuencia, la pandemia y la crisis económica que la acompaña, incluida la pérdida masiva de empleos a nivel mundial, pondrán a los niños en situación de pobreza en un riesgo aún mayor, exacerbando, en gran medida, las desigualdades existentes.

Finalmente, en relación con la salud mental de NNA, eventos disruptivos como la pandemia y el posterior estado de confinamiento, inevitablemente tendrán efectos en la salud mental de la población, dado que se están enfrentando, a nivel individual y familiar, situaciones de estrés e incertidumbre generadas por los abruptos cambios en las rutinas como consecuencia del cierre de escuelas y jardines infantiles. A su vez, el exceso de noticias que circulan en diferentes medios de comunicación y redes sociales expone a la población infantil a información que puede afectarla emocionalmente cuando no es debidamente acompañada y procesada en compañía de un adulto. En este contexto, es esperable que se produzcan respuestas adaptativas tales como miedo, ansiedad, irritabilidad, angustia y enojo (Minsal, 2020). 
Cuando lo anterior se combina con la angustia que gatilla la preocupación por enfermarse o que miembros de la familia se contagien o mueran, la percepción de la preocupación de los padres o familiares por la posible pérdida de empleos y el aumento de las tensiones dentro de los hogares, pueden producirse sentimientos de impotencia y una mayor vulnerabilidad que pueden deteriorar la salud mental (Unicef, 2020). En este ámbito es importante señalar que los padres y los cuidadores primarios también se verán afectados y necesitarán un acompañamiento que los considere en su sufrimiento y afectación. Se trata, en definitiva, de una aproximación que considere al niño, su familia y su contexto desde un abordaje psicosocial:

La salud mental no es solo ausencia de enfermedad, sino también es bienestar subjetivo, es decir, la satisfacción y capacidad de desarrollo respecto de los proyectos de vida. Por tanto, el tema debe abordarse no solo médicamente, sino psicosocialmente (Universidad de Chile, 2020, p. 3).

La salud mental es un elemento clave y estratégico para hacer frente a las consecuencias de la crisis sociosanitaria en el mediano y largo plazo en lo que respecta a la preservación y reconstrucción del tejido social, siempre y cuando se considere el diseño de una política que se articule de forma participativa entre diferentes actores, lo que incluye a los NNA, considerando dimensiones territoriales e intersectoriales para la implementación y seguimientos de medidas y planes de intervención.

En definitiva, el impacto de la pandemia en NNA se extiende mucho más allá de los efectos en la salud física y se da en un escenario donde la situación de la infancia en nuestro país ya se encontraba en un estado de crisis, considerando la ausencia de una Ley de Garantías de los Derechos de la Niñez, la falta de reconocimiento constitucional, los alarmantes índices de violencia y maltrato que reportan los propios niños (Unicef, 2015), y las graves y sistemáticas vulneraciones de derechos en residencias de protección, como ha señalado el Comité de los Derechos del Niño de Naciones Unidas (2018).

La magnitud de los efectos del Covid-19 puede aumentar cuando se presentan factores de riesgo como los estigmas, la discriminación, la desigualdad entre los géneros, los entornos superpoblados, la pobreza, la pérdida de empleo y acceso a servicios de salud, todos fenómenos que dejan principalmente a los niños expuestos a una mayor vulnerabilidad y angustia psicosocial, como señala la nota técnica elaborada por The Alliance for Child Protection in Humanitarian Action (2020).

Por otra parte, uno de los mayores desafíos que instala esta pandemia son las acciones de prevención y respuestas que las sociedades y los gobiernos deberán adoptar a medida que se vayan levantando las cuarentenas y se avizore la posibilidad 
de retorno a los espacios laborales y educacionales asegurando la protección de las personas. Probablemente, será necesario elaborar estrategias dinámicas que consideren la entrada y salida de cuarentenas, así como incorporar en una nueva cotidianidad los confinamientos.

En este punto cabe preguntarse: ¿cómo será la vida después de la pandemia? ¿Cómo pueden las personas y las comunidades prepararse para posibles rebrotes o futuras pandemias? ¿Cómo se protegerán y garantizarán los derechos de NNA considerando su bienestar, desarrollo integral y sus voces en este tipo de acontecimientos?

\section{CUARENTENA Y CONFINAMIENTO. EFECTOS Y SIGNIFICADOS}

Una de las experiencias más significativas que han vivido los NNA durante la pandemia son las situaciones de cuarentenas voluntarias y obligatorias como medidas que los gobiernos imponen a los ciudadanos para evitar la propagación del virus en la población. Esta experiencia es relevante para analizar los efectos de la crisis sociosanitaria en la salud mental de los niños, ya que permite reflexionar sobre la paradoja que se inscribe en este mecanismo de salud pública para la prevención de epidemias. Se trata de una medida que tiene por objeto proteger la vida biológica, pero que produce una serie de consecuencias en la vida afectiva y social de las personas, particularmente de los NNA.

Según el Center for Disease Control and Prevention (2017), la cuarentena es un concepto que se define como la separación y restricción de movimientos de personas que estuvieron expuestas a una enfermedad infecciosa, pero que no tienen síntomas, para observar si desarrollan la enfermedad. Se diferencia del aislamiento, que es la separación de personas que padecen una enfermedad contagiosa de aquellas que están sanas. Ambas medidas son estrategias de salud pública que tienen como objetivo prevenir la propagación de enfermedades infecciosas.

Existen registros históricos de aplicación de cuarentenas y aislamientos ya en el Nuevo Testamento, así como en Grecia y el Imperio Romano en los siglos V y VI a.C. Sin embargo, la cuarentena se origina formalmente en el siglo XIV, en Italia, como una medida para controlar las epidemias de peste negra que azotaban a Europa y que obligaba a los barcos y personas que provenían de Asia a esperar 40 días ("quaranta giorni", en italiano) antes de entrar en las ciudades, tras comprobar que no estaban enfermos:

La cuarentena nació en 1374, con el edicto de Reggio, ciudad de Módena, Italia. En realidad, fue un cordón sanitario, pues el término cuarentena derivó en término marítimo, aplicándose un período de aislamiento a los buques que llegaban de 
puertos de mala fama médica. Este período llevaba implícita la idea del período de incubación. El primer puerto en que se decretó cuarentena (que fue sólo treintena: luego se ampliaría) fue Ragusa (hoy Dubrovnik, Bosnia-Herzegovina, sobre el Adriático) en 1377. Seis años después, Marsella aumentó el plazo a los cuarenta días. En el siglo XV este período de observación o cuarentena hizo nacer el lazareto, también en Marsella, 1476, lugar complementario donde los pasajeros debían permanecer en espera que pasase el período de contagio arbitrariamente establecido (Ledermann, 2003, p. 15).

Estos antecedentes históricos son relevantes para situar el origen de estas medidas y las consecuencias que fueron produciendo en las condiciones de vida de la población, así como en sus actividades cotidianas. Actualmente, la duración de la cuarentena se define según el periodo de incubación de la enfermedad. En el caso del Covid-19, ese periodo comprende 14 días.

Las cuarentenas, en tanto medidas de salud pública, tienen una serie de implicancias en las experiencias individuales y colectivas de las poblaciones. Una de ellas es la imposición externa que restringe la libertad de acción y movimiento. Esta experiencia está asociada a sintomatología depresiva y de estrés postraumático; se vive con desagrado, ya que implica la separación de los seres queridos, la pérdida de la libertad, la incertidumbre sobre la situación de la enfermedad y el aburrimiento (Brooks et al., 2020). Por lo tanto, los eventuales beneficios de la implementación de estas estrategias deben ser sopesados cuidadosamente, considerando los posibles costos psicológicos y sociales.

La revisión sistemática realizada por Brooks y colaboradores (2020) establece que dentro de los principales factores estresores que podemos encontrar durante los confinamientos se pueden destacar los siguientes: la duración de la cuarentena; el miedo a enfermarse o a que algún ser querido se contagie; la frustración como consecuencia de la pérdida de las rutinas; la falta de suministros básicos para alimentarse; no disponer de información adecuada, oportuna y transparente por parte de la autoridades; problemas financieros y la estigmatización por parte de otros miembros de la comunidad.

\section{EL ESTUDIO CONCLUYE:}

La cuarentena es una de varias medidas de salud pública para prevenir la propagación de una enfermedad infecciosa y como se muestra en revisión, tiene un considerable impacto psicológico para los afectados. Por lo tanto, hay una pregunta en cuanto a si otras medidas de salud pública que impiden la necesidad 
de imponer una cuarentena (como el distanciamiento social, cancelación de reuniones masivas y cierres de escuelas) podrían ser más favorables (...) El impacto de la cuarentena es amplio, sustancial y puede ser de larga duración. Esto no es para sugerir que la cuarentena no debe utilizarse; los efectos psicológicos de no utilizar cuarentena y permitir la propagación de la enfermedad podrían ser peores. Sin embargo, privar a las personas de su libertad por el bien público en general es a menudo polémico y debe ser manejado con cuidado. Si la cuarentena es esencial, nuestros resultados sugieren que los funcionarios deben tomar todas las medidas para asegurar que esta experiencia sea lo más tolerable posible para la gente (Brook et al., 2020, p. 919).

Por otra parte, una investigación desarrollada por Orgilés y colaboradores (2020) realizó una encuesta a 1.143 padres y cuidadores de niños italianos y españoles entre 3 y 18 años, quienes completaron una encuesta que proporcionó información sobre la forma en que la cuarentena afecta a sus hijos y a ellos mismos, estableciendo una comparación con su situación previa a la cuarentena. Dentro de las principales conclusiones se puede destacar que el 85,7\% de los padres informaron de cambios en el estado emocional y el comportamiento de sus hijos durante la cuarentena. El síntoma más frecuente fue la dificultad de concentración, reportado por el $76,6 \%$ de los padres. El aburrimiento, la irritabilidad, la inquietud, el nerviosismo, los sentimientos de soledad, la intranquilidad y una mayor preocupación fueron reportados por más del 30\% de los padres. Se concluye, además, que habría una relación entre los cambios observados y la situación emocional de los padres:

Los síntomas emocionales y de comportamiento de los niños de ambos países parecen estar positivamente relacionados con el bienestar de los padres, específicamente con su nivel de estrés. Sobre la base de estos hallazgos, podemos concluir que tanto los niños como los padres están afectados por una situación tan estresante como la cuarentena (Orgilés et al., 2020, p. 11).

Desde un punto de vista de las percepciones y significados que los propios NNA han construido en relación a la situación de confinamiento, el estudio exploratorio de Martínez, Rodríguez y Velázquez (2020) entrega información muy relevante sobre las experiencias de la niñez a través de una metodología que recoge sus voces y opiniones.

Mediante un cuestionario en línea se recopiló el universo vivencial de más de 400 participantes de diferentes ciudades de España con el propósito de recoger de primera mano las voces de niños y niñas como participantes esenciales de la vida 
cotidiana. Por lo tanto, la aproximación metodológica supone la idea de que las y los niños son actores sociales, integrantes de una comunidad y que sin ellos no se puede comprender la sociedad y sus transformaciones.

Uno de los resultados que se puede destacar del estudio son los significados que NNA proponen para una definición de confinamiento desde sus propias formas de habitar la realidad. Desde sus opiniones, este se configura como una experiencia de encierro que se vive con resignación y cuyo principal efecto es renunciar al modo habitual para sostener las relaciones sociales. A su vez, esta situación tiene un significado de solidaridad intergeneracional, ya que supone que esta forma de cuidado implica un bien mayor para la comunidad (Martínez et al., 2020).

Otra conclusión relevante tiene relación con los principales sentimientos que los NNA declaran haber tenido durante la cuarentena, entre los que destaca la sensación de tranquilidad. En tanto, con relación a las sensaciones con potencial perturbador, el aburrimiento y la preocupación son las que surgen con mayor frecuencia.

Esta aproximación no solo permite recoger las experiencias y significados que los propios NNA construyen durante la pandemia, sino que además visibiliza a los niños como reproductores y productores de cultura en un escenario que no ha considerado su participación ni la relevancia de sus puntos de vista para una comprensión más amplia e inclusiva de la situación de crisis sociosanitaria.

En general, la discusión sobre los efectos de la pandemia en los NNA y las consecuencias en su bienestar y desarrollo han prescindido de sus percepciones, narrativas y emociones. Son más bien constructos cuyos contenidos están dados desde discursos hegemónicos que suponen e interpretan a los niños sin considerar sus experiencias, producciones y lógicas para adaptarse y transitar esta nueva cotidianidad.

Lo señalado hasta este punto es de gran importancia, ya que se relaciona con un paradigma conceptual de la niñez que condiciona y fija el rol de los NNA exclusivamente a un estado de vulnerabilidad, y que en un estado de emergencia como el de la pandemia, amenaza con dejarlos excluidos de participar activamente de la vida ciudadana y política en un momento de profundos cambios sociales y del modo de vida.

La infancia no es una categoría homogénea, por lo que el grado de afectación y las formas de significación de las experiencias estarán asociados a factores como edad, género, nivel socioeconómico, localización geográfica y etnia. En ese plano, es relevante observar en los NNA las modalidades de adaptación, sostén y producción de los vínculos sociales, y a la vez identificar aquellos elementos que obstaculizan los intercambios afectivos, la organización autónoma de grupos de pares, la construcción de nuevas identidades y los lazos sociales como modalidades que permiten ir tejiendo nuevas formas de colectividad. 


\section{REFLEXIONES FINALES}

Los efectos del confinamiento en la niñez y sus implicancias en la salud mental corresponden a un ámbito que plantea principalmente preguntas sobre las particularidades de las experiencias infantiles en estas nuevas circunstancias sociales, reconociendo sus recursos y estrategias de adaptación, así como las formas y causas del malestar psíquico que pueden producir estas experiencias. ¿De qué manera han cambiado las vidas de niños, niñas y adolescentes a partir del confinamiento? ¿Qué mecanismos de adaptación se producen en estos contextos? ¿Qué efectos tiene el repliegue que va desde el espacio público a la intimidad de la vida familiar? Son estas algunas preguntas que pueden invitar a seguir reflexionando sobre este acontecimiento histórico en el territorio de la infancia.

A partir de los antecedentes presentados, es posible señalar que para los NNA la cuarentena es una experiencia que implica el abandono y repliegue masivo de los espacios públicos que configuraban, hasta hace muy poco, una parte fundante de su cotidianidad: jardines infantiles, escuelas, colegios, calles, plazas y parques eran espacios de intercambio y socialización con otros niños y adultos. En ese sentido, la cuarentena constituye un fenómeno que provoca la separación de los lazos afectivos y la discontinuidad de la vida cotidiana, y que irrumpe abruptamente en las rutinas y vínculos con otros significativos como pares, familiares y otros adultos.

Desde esa perspectiva, las cuarentenas y confinamientos son acontecimientos que pueden producir un corte radical en las experiencias de continuidad y proximidad de un NNA en el campo de las relaciones afectivas, los vínculos significativos, los grupos de pertenencia y en lo que refiere a los espacios de intimidad y diferenciación del grupo familiar.

Siguiendo la misma lógica, pero en un sentido inverso, las experiencias de separación producidas por las cuarentenas tienen como antecedente histórico el estudio de los efectos devastadores observados en la evacuación masiva de niños de ciudades europeas durante la Segunda Guerra Mundial y su posterior retorno. En ese contexto, la discontinuidad y la pérdida de los vínculos primarios generó importantes hallazgos sobre la relevancia que tienen para un niño sus relaciones afectivas y la manera en que la ruptura de esos vínculos conlleva efectos negativos en su experiencia vital.

Una pregunta que se puede articular en este contexto y que remite a los acontecimientos de la Segunda Guerra Mundial es ¿por qué los niños y sus padres deben pagar un precio tan alto como los graves problemas de salud mental y la pérdida de los vínculos sociales y afectivos derivados de la separación y el confinamiento, para protegerse del contagio? En 1939, el pediatra y psicoanalista Donald Winnicott se hacía el mismo cuestionamiento: 
Si uno intenta colocarse en el lugar de la madre, se plantea de inmediato esta pregunta: ¿por qué, en realidad, se aleja a los niños del riesgo de los ataques aéreos a un precio tan alto y causando tantas dificultades? ¿Por qué se pide a los padres que hagan semejante sacrificio? (Winnicott, 1939, p. 45).

Por lo tanto, la situación de confinamiento nos debe llevar a reflexionar sobre el lugar de los vínculos durante la pandemia, considerando que la pérdida de los lazos afectivos y sociales es un asunto que puede afectar profundamente la experiencia humana y traer consecuencias para la salud mental de los individuos y comunidades.

El confinamiento implica resignar una forma de relación con el otro, pérdida de la posibilidad de encuentros, intercambios y formas de construir relaciones y comunidad en momentos donde se vuelve necesario reconstruir el tejido social sobre la base de la confianza y el reconocimiento del otro en un plano de responsabilidad y en un proyecto de sociedad colectivo.

Pero el confinamiento también introduce un problema relacionado con los intercambios intergeneracionales y el proceso de historización como vías que permiten el registro y la memoria. La repetición totalizante de una vida cotidiana que se cierra sobre sí misma es también una amenaza para hacer memoria de una experiencia vital que, sumida en la inercia de una cotidianidad repetitiva y a la vez estresante, puede terminar resignando la capacidad para desear, crear y compartir con otros.

Reconocer y dar lugar a las experiencias y a las nuevas formas de vinculación de la infancia es un paso necesario para cuidar y proteger los derechos, bienestar y desarrollo de los NNA, quienes deben ser reconocidos en su dimensión ciudadana y política y no solo como receptores pasivos de las medidas gubernamentales para enfrentar la pandemia. Sin esa inclusión a los procesos de transformación social, la infancia quedará excluida de participar y, más grave aún, quedará vulnerable frente a un sistema que no le reconoce su capacidad de agencia y generación de cambios.

La pandemia de Covid-19 no solo ha puesto de relieve graves deficiencias en la protección de los niños de muchos países y la insuficiencia de los sistemas de educación, atención de salud y protección social, sino que también muestra las brechas de inclusión y reconocimiento de los niños como sujetos de pleno derecho. Una respuesta a la crisis de Covid-19 que respete los derechos de los NNA no debe mitigar únicamente los peores daños de la pandemia, sino que también debe considerar una nueva forma de relación entre la infancia, la sociedad y el Estado, respetuosa de sus experiencias, procesos y formas de organización. 


\section{REFERENCIAS}

Beloff, M. (1999). Modelo de la protección integral de los derechos del niño y de la situación irregular: un modelo para armar y otro para desarmar. Justica y Derechos del Niño; 1: 9-21.

Brooks, S. K., Webster, R. K., Smith, L. E. et al. (2020). The psychological impact of quarantine and how to reduce it: Rapid review of the evidence. Lancet. 2020;395(10227):912-920.

Center for Disease Control and Prevention. Quarantine and isolation. 2017. Recuperado de: https://www.cdc.gov/quarantine/index.html

Centro de Investigaciones de Unicef (2017). Construir el futuro: Los niños y los Objetivos de Desarrollo Sostenible en los países ricos. Report Card N 14 de Innocenti, Centro de Investigaciones de Unicef, Florencia.

Clark, H., Coll-Seck, A. M., Banerjee, A. et al. (2020). A future for the world's children? A WHO- Unicef-Lancet Commission. Lancet 2020; 395: 605-58.

Comisión Interamericana de Derechos Humanos (2020). Pandemia y derechos humanos en las Américas. Resolución 1/2020. Recuperado de: https://www.oas.org/es/ cidh/decisiones/pdf/Resolucion-1-20-es.pdf

Comité de los Derechos del Niño (2018). Informe de la investigación relacionada en Chile en virtud del artículo 13 del Protocolo Facultativo de la Convención sobre los Derechos del Niño relativo a un procedimiento de comunicaciones. Recuperado de: http://www. minjusticia.gob.cl/media/2018/07/Informe_del_Comit\%C3\%A9_de_la_ Ni\%C3\%B1ez_de_ONU.pdf

Comité de los Derechos del Niño (2020). Declaración sobre el grave efecto físico, emocionaly psicológico de la pandemia Covid-19 en los niños. Recuperado de: https:// tbinternet. ohchr.org/Treaties/CRC/Shared\%20Documents/1_Global/INT_CRC_ STA_9095_S.pdf

Durkheim, É. (2001). Las reglas del método sociológico. Madrid: Akal.

Human Rights Watch (2020). Covid-19 and Children's Rights. Recuperado de: https:/ / www.hrw.org/news/2020/04/09/covid-19-and-childrens-rights-0

Ledermann, W. (2003). El hombre y sus epidemias a través de la historia. Revista Chilena de Infectología, 20, 13-17. http:/ /dx.doi.org/10.4067/S0716-10182003020200003

Martínez, M., Rodríguez, I., Velásquez, G. (2020). Infancia confinada. ¿Cómo viven la situación de confinamiento niñas, niños y adolescentes? Madrid. 
Minsal (2020). Guía práctica de bienestar emocional: cuarentena en tiempos de Covid-19. Recuperado de: https://www.minsal.cl/wp-content/ uploads/2020/04/2020.04.12_GUIA-PRACTICA-CUARENTENA-ENTIEMPOS-DE-COVID19_final.pdf?fbclid=IwAR38yy6BCFWGpzhW3Z_ xqPmHIXm1LBY9J4adPeYMNfrUPUAnhNMrtPsY3UM

Orgilés, M., Morales, A., Delveccio, E. et al. (2020). Immediate psychological effects of the Covid-19 quarantine in youth from Italy and Spain. Recuperado de: https://psyarxiv.com/qaz9w/

The Alliance for Child Protection in Humanitarian Action, Technical Note: Protection of Children during the Coronavirus Pandemic, Version 2, May 2020.

The Alliance for Child Protection in Humanitarian Action, End Violence Against Children, Unicef, WHO, Covid-19: Protecting Children from Violence, Abuse and Neglect in the Home, Version 1, May 2020.

Unicef (2015). $4^{\circ}$ Estudio de maltrato infantil en Chile. Recuperado de: https://www. unicef.org/chile/media/1306/file/4to_estudio_de:maltrato_infantil_en_ chile.pdf

Unicef (2016). Ending extreme poverty: a focus on children. Recuperado de: https://www.unicef.org/reports/ending-extreme-poverty-focus-children

Universidad de Chile (2020). Salud mental en situación de pandemia: documento para mesa social Covid-19. Recuperado de: https://www.uchile.cl/ noticias/162386/rector-presenta-a-mesa-social-por-covid-19-propuesta-desalud-mental

United Nations (2020). Policy brief: impact of Covid-19 on children. Recuperado de: https://unsdg.un.org/sites/default/files/202004/160420_Covid_ Children_Policy_Brief.pdf

Winnicott, D. (2009). El retorno del niño evacuado. En Deprivación y Delincuencia. Buenos Aires: Paidós. 